\title{
Threshold of Terahertz Population Inversion and Negative Dynamic Conductivity in Graphene Under Pulse Photoexcitation
}

\author{
A. Satou . V. Ryzhii, Y. Kurita, and T. Otsuji \\ Research Institute of Electrical Communication, Tohoku University, \\ 2-1-1 Katahira, Aoba-ku, Sendai 980-8577, Japan
}

(Dated: October 29, 2012)

\begin{abstract}
We theoretically study the population inversion and negative dynamic conductivity in intrinsic graphene in the terahertz $(\mathrm{THz})$ frequency range upon pulse photoexcitation with near-/mid-infrared wavelength. The threshold pulse energy required for the population inversion and negative dynamic conductivity can be orders-of-magnitude lower when the pulse photon energy is lower, due to the inverse proportionality of the photoexcited carrier concentration to the pulse photon energy and to the weaker carrier heating. We also investigate the dependence of the dynamic conductivity on the momentum relaxation time. The negative dynamic conductivity takes place either in high- or low-quality graphene, where the Drude absorption by carriers in the $\mathrm{THz}$ frequency is weak.

PACS numbers: 78.47.J-, 78.45.+h, 78.20.Bh, 81.05.ue
\end{abstract}

\section{INTRODUCTION}

Graphene has attracted much attention for wide variety of device applications due to its exceptional electronic and optical properties $\underline{\underline{1}-\underline{3}}$ Especially, $\mathrm{THz}$ devices such as lasers ${ }^{4-9}$ and photodetectors ${ }^{10}-12$ utilizing its high carrier mobility and gapless dispersion have been investigated (for a review of these devices, see Ref. 13). In Refs. 4 - 8 , we demonstrated that the population inversion can occur in optically pumped graphene at $\mathrm{THz} /$ far-infrared range of frequency and hence the lasing at such a range is possible, utilizing the gapless linear energy spectrum and relatively high optical-phonon (OP) energy in graphene. Due to the energy spectrum $\varepsilon= \pm v_{F} \hbar k$, where $v_{F} \simeq 10^{8}$ $\mathrm{cm} / \mathrm{s}$ is the Fermi velocity and $k$ is the wavenumber, the Fermi energy $\varepsilon_{F}$ in equilibrium in intrinsic graphene is equal to zero. Hence, the electron and hole distribution functions at the bottom of the conduction band and the top of the valence band have values $f_{\mathrm{e}}(0)=f_{\mathrm{h}}(0)=1 / 2$. This implies that upon photoexcitation one might expect to make the values of the distribution functions at low energies greater than one half, i.e., $f_{\mathrm{e}}(\varepsilon)=f_{\mathrm{h}}(\varepsilon)>1 / 2$, corresponding to the population inversion. Such a population inversion leads to the total dynamic conductivity in graphene at $\mathrm{THz} /$ far-infrared frequencies being negative, and lasing in graphene at such frequencies is possible.

Recently, we measured the carrier relaxation and recombination dynamics in optically pumped epitaxial graphene on silicon 14 and exfoliated graphene $\frac{15}{\underline{15}}$ using $\mathrm{THz}$ time-domain spectroscopy based on an optical pump/THz\&optical probe technique, and observed the amplification of $\mathrm{THz}$ radiation by stimulated emission from graphene under pulse excitation. To our knowledge, those are the first observation of the $\mathrm{THz}$ amplification using optically pumped graphene. Those results demonstrate the possibility of realizing $\mathrm{THz}$ lasers based on graphene.

The carrier dynamics in optically pumped graphene strongly depends on the initial temperature of carriers and the intensity of the optical pumping. With suffi- ciently low carrier concentration, i.e., at low temperature and upon weak pumping, photoexcited carriers are effectively accumulated near the Dirac point via cascade emission of OPs, and the achievement of the population inversion is expected to be efficient $\underline{4,5,8}$ On the contrary, at room temperature or upon not so weak pumping where the carrier concentration is rather high, the carrier-carrier (CC) scattering plays a crucial role in the dynamics after the pulse excitation, due to fast quasi-equilibration of carriers. In fact, ultrafast optical pump-probe spectroscopy on graphene has indicated that the quasi-equilibration by the CC scattering takes place within the time scale of $10 \mathrm{fs}, \underline{\underline{16}-\underline{19}}$ which is much faster than the process of single OP emission. In such a case, the pulse excitation makes carriers very hot initially, and the energy relaxation and recombination via OP emission follow.

Previously, we studied the population inversion and the negative dynamic conductivity in intrinsic graphene at room temperature upon the pulse excitation with photon energy $0.8 \mathrm{eV}$, corresponding to the wavelength 1.55 $\mu \mathrm{m}$, and showed that they can be achieved with the pulse energy fluence above the threshold $: \underline{7.20}$

In this paper, we investigate the dependence of the threshold pulse fluence for the $\mathrm{THz}$ negative dynamic conductivity on the pulse photon energy, using the model extended from that developed in Refs. 7 and 20 to take into account the effect of the Pauli blocking. We also study the dependence of the dynamic conductivity on the momentum relaxation time $\tau$. We consider the two limiting cases where $\omega \tau \geq 1$, corresponding to highquality graphene, and where $\omega \tau \ll 1$, corresponding to low-quality graphene.

\section{EQUATIONS OF THE MODEL}

Assuming the quasi-Fermi distribution of carriers due to the quasi-equilibration by the $\mathrm{CC}$ scattering, i.e., $f_{\varepsilon}=\left\{1+\exp \left[\left(\varepsilon-\varepsilon_{F}\right) / k_{B} T_{c}\right]\right\}^{-1}$, the time evolution of 
the distribution is represented through the quasi-Fermi level $\varepsilon_{F}(t)$ and the carrier temperature $T_{c}(t)$. Here, both electron and hole distributions can be expressed by a single "carrier distribution" because we consider intrinsic graphene; due to the symmetric dispersion for electrons and holes in the energy range under consideration, i.e., $\varepsilon<1 \mathrm{eV}$, their distributions remain identical even upon the pulse excitation. The rate equations which determine the quasi-Fermi level and carrier temperature can be obtained from the quasi-classical Boltzmann equation: $:, 20,21$

$$
\begin{aligned}
\frac{d n}{d t}= & \frac{2}{\pi} \sum_{i=\Gamma, \mathrm{K}} \int_{0}^{\infty} k d k \times \\
& {\left[\frac{\left(1-f_{\hbar \omega_{i}-\varepsilon}\right)\left(1-f_{\varepsilon}\right)}{\tau_{i, \text { inter }}^{(+)}}-\frac{f_{\varepsilon} f_{\hbar \omega_{i}-\varepsilon}}{\tau_{i, \text { inter }}^{(-)}}\right], } \\
\frac{d \mathcal{E}}{d t}= & \frac{2}{\pi} \sum_{i=\Gamma, \mathrm{K}} \int_{0}^{\infty} k d k \times \\
& \left\{\hbar \omega_{i}\left[\frac{f_{\varepsilon}\left(1-f_{\varepsilon+\hbar \omega_{i}}\right)}{\tau_{i, \text { intra }}^{(+)}}-\frac{f_{\varepsilon}\left(1-f_{\varepsilon-\hbar \omega_{i}}\right)}{\tau_{i, \text { intra }}^{(-)}}\right]\right. \\
+ & {\left[\frac{\left.\left(1-f_{\left.\hbar \omega_{i}-\varepsilon\right)\left(1-f_{\varepsilon}\right)}^{(+)}-\frac{f_{\varepsilon} f_{\hbar \omega_{i}-\varepsilon}}{\tau_{i, \text { inter }}^{(-)}}\right]\right\},}{\text {inter }}\right.}
\end{aligned}
$$

where $n=n\left(\varepsilon_{F}, T_{c}\right)$ and $\mathcal{E}=\mathcal{E}\left(\varepsilon_{F}, T_{c}\right)$ are the concentration and energy density of either type of carriers, which are found by integrating over $\boldsymbol{k}$ the distribution function multiplied by proper factors, the index $i$ runs over types of OPs (long-wavelength $\Gamma$-OP and short-wavelength $K$ $\mathrm{OP})$, and $\tau_{i, \text { intra }}^{( \pm)}$and $\tau_{i, \text { inter }}^{( \pm)}$are the intraband and interband scattering rates for OPs $((+)$ for absorption and $(-)$ for emission). Those rates are on the order of subpicoseconds for high-energy carriers.

Note that the first term (intraband transition) in the curly brackets in Eq. (2) contains the factor $\hbar \omega_{i}$, whereas the second term (interband transition) contains $\varepsilon$. These are energy that a carrier looses/acquires in their corresponding transitions. The energy conservation for the latter transition is implicitly satisfied because the rest of the OP energy $\hbar \omega_{i}-\varepsilon$ is taken into account in the same equation for the other kind of carrier. Here, we assume the equilibrium OPs and neglect the nonequilibrium population of OPs due to their emission via OP scattering of carriers. The effects of the latter have been discussed elsewhere $\underline{\underline{8}}$

Equations (11) and (2) together with initial conditions form a nonlinear system of equations for $\varepsilon_{F}(t)$ and $T_{c}(t)$, which can be solved numerically. Equations (11) and (2) are accompanied with the initial carrier concentration and energy density from which the initial quasi-Fermi level and carrier temperature can be found:

$$
\left.n\right|_{t=0}=n_{0}+\Delta n,\left.\quad \mathcal{E}\right|_{t=0}=\mathcal{E}_{0}+\Delta \mathcal{E},
$$

where $n_{0}$ and $\mathcal{E}_{0}$ are the intrinsic carrier concentration and energy density, and $\Delta n$ and $\Delta \mathcal{E}$ are contributions of photogenerated carriers. Those are equal to the concentration and energy density generated from the pumping:

$$
\Delta n \simeq \frac{\pi \alpha \Delta J}{\sqrt{\epsilon} \hbar \Omega}\left(1-\left.2 f_{\hbar \Omega / 2}\right|_{t=0}\right), \quad \Delta \mathcal{E} \simeq \frac{\hbar \Omega}{2} \Delta n,
$$

where $\alpha \sim 1 / 137, \epsilon$ is the dielectric constant surrounding graphene, $\hbar \Omega$ and $\Delta J$ are the pulse photon energy and the pulse fluence.

In Eq. (4), the last factor $1-2 f_{\hbar \Omega / 2}$ roughly takes into account the Pauli blocking of the photoabsorption, and the distribution function in it is approximated by the quasi-Fermi distribution after the photoabsorption with $\left.\varepsilon_{F}\right|_{t=0}$ and $\left.T_{c}\right|_{t=0}$ calculated self-consistently from Eqs. (3) and (4). This approximation underestimates the effect of the Pauli blocking by the nonequilibrium photogenerated carriers when the pulse is so intense that the rate of the photogeneration is comparable to that of the quasi-equilibration by the carrier-carrier scattering. On the other hand, it overestimates the effect by timedependent increase in the distribution function during the pulse excitation, somewhat compensating the underestimation described above. In addition, we assume the pulse has sufficiently short width that the OP scattering during the absorption of the pulse is not effective, i.e., $\Delta t \lesssim \tau_{i, \text { inter }}^{(+)}(\hbar \Omega / 2), \tau_{i, \text { intra }}^{(+)}(\hbar \Omega / 2)$.

To study not only the gain acquired by the population inversion but also the net gain taking into account the loss due to the Drude absorption of electromagnetic waves by carriers in graphene, we introduce the real part of dynamic conductivity ${ }^{22}$ as it is related to the net gain:

$$
\begin{aligned}
\operatorname{Re} \sigma_{\omega} \simeq & \frac{e^{2}}{4 \hbar}\left(1-2 f_{\hbar \omega / 2}\right) \\
& +\frac{2 e^{2} v_{F}^{2}}{\pi} \int_{0}^{\infty} d k k \frac{\tau}{1+(\omega \tau)^{2}}\left(-\frac{d f_{\varepsilon}}{d \varepsilon}\right),
\end{aligned}
$$

where $\tau$ is the momentum relaxation time. The first term in Eq. (5) corresponds to the interband contribution which depends on the frequency only through the distribution function and which can be negative when the rate of stimulated emission exceeds the rate of absorption, i.e., when the population inversion takes place. Since the distribution function is the quasi-Fermi distribution, the condition of the population inversion for the photon energy $\hbar \omega$ is simply represented as $\varepsilon_{F}>\hbar \omega / 2$. On the other hand, the second term corresponds to the intraband contribution (Drude conductivity) which is always positive in the system under consideration.

When the real part of the dynamic conductivity becomes negative at some frequency, the electromagnetic wave at that frequency passing through optically pumped graphene is amplified and it serves as a gain medium. Note that from Eq. (5) the largest achievable negative value of the conductivity is $-e^{2} / 4 \hbar$, corresponding to the amplification of $(2.3 / \sqrt{\epsilon}) \%$ of the incoming electromagnetic wave. 
(a) $\hbar \Omega=0.8 \mathrm{eV}$

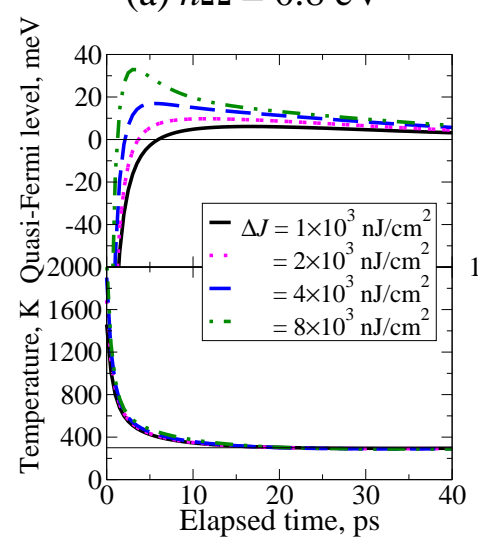

(c) $\hbar \Omega=0.2 \mathrm{eV}$

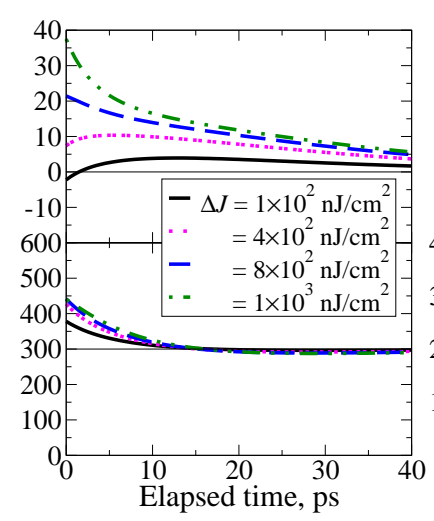

(b) $\hbar \Omega=0.4 \mathrm{eV}$

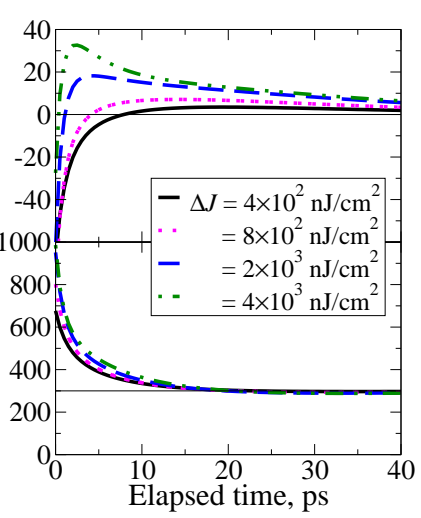

(d) $\hbar \Omega=0.1 \mathrm{eV}$

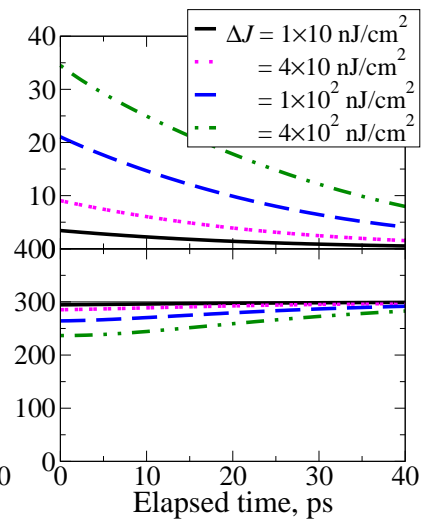

FIG. 1. The time evolution of quasi-Fermi level and carrier temperature with different pulse fluence and pulse photon energy $[(\mathrm{a}) \hbar \Omega=0.8 \mathrm{eV}$, (b) $0.4 \mathrm{eV}$, (c) $0.2 \mathrm{eV}$, (d) $0.1 \mathrm{eV}]$. Room temperature $(300 \mathrm{~K})$ is indicated by thin solid lines.

\section{RESULTS AND DISCUSSION}

\section{A. Time Evolution of Quasi-Fermi Level and Carrier Temperature}

Using the model above, we investigate the population inversion and the time-dependent dynamic conductivity in intrinsic graphene at room temperature after pulse excitation. We varied the pulse photon energy $\hbar \Omega$ in the range between $0.1-0.8 \mathrm{eV}$ corresponding to infrared wavelength of $12.4-1.55 \mu \mathrm{m}$. Besides, we set $\epsilon=5.5$ corresponding to the effective dielectric constant of the interface between the air and SiC. Here, we have used the pulse fluence rather than the pulse intensity as a measure of pulse strength because the former does not depends explicitly on the pulse photon energy, whereas the latter does through the pulse width.

Figure 1 shows the time evolution of the quasi-Fermi level and carrier temperature after the pulse excitation with different pulse fluence and pulse photon energy. It is clearly demonstrated in Fig. [1 that the population in-

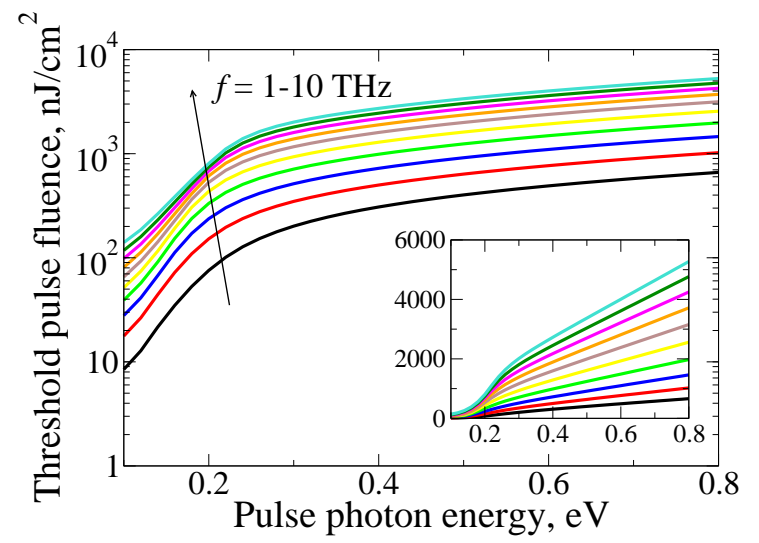

FIG. 2. The dependence of the threshold fluence of the population inversion on the pulse photon energy for different frequencies of $\mathrm{THz}$ photons. The inset shows the same dependence but with linear scale.

version at $\mathrm{THz}$ frequencies, say, up to about $10 \mathrm{THz}$ corresponding to $\hbar \omega / 2 \sim 20 \mathrm{meV}$ is achieved with some threshold pulse fluence, and that it lasts on the order of 10 ps.

The mechanism of the population inversion in the $\mathrm{THz}$ range is explained as follows. Right after the pulse excitation, the carrier temperature becomes very hot (except for the excitation with low photon energy) and the quasi-Fermi level can even be negative due to the quasiequilibration of carriers with very large heat brought by the photogenerated carriers. After a few ps, carriers with high energy are relaxed via intraband OP emission and accumulated in the low energy region, which is illustrated by the increase in the quasi-Fermi level. As discussed in Ref. 20, the population inversion lasts on the order of 10 ps due to the imbalance between the time scales of intraand inter-band OP emission.

Comparing Figs. 1(a)-(d), one can see that the threshold fluence of the population inversion becomes lower as the pulse photon energy becomes lower. This is also seen in Fig. 2 that illustrates the dependence of the threshold fluence of the population inversion on the pulse photon energy for different frequencies of $\mathrm{THz}$ photons. There are several factors for this lowering. First, the lower fluence is needed for the pulse with lower photon energy to have the same amount of carrier concentration [more precisely, $\Delta J \propto \hbar \Omega$, as seen in Eq. (44)]. Second, the heating of carriers becomes less and the carrier temperature becomes lower [even the cooling can occur as shown in Fig. 1(d); see below for more details], resulting in their denser accumulation in the low energy region. Finally, the interband OP emission, i.e., the recombination is suppressed due to the lower carrier temperature. All these result in the nonlinear lowering of the threshold as the pulse photon energy decreases; Fig. 2 exhibits almost linear dependence of the threshold in the region of large photon energy, caused by the first factor, and the sharp 


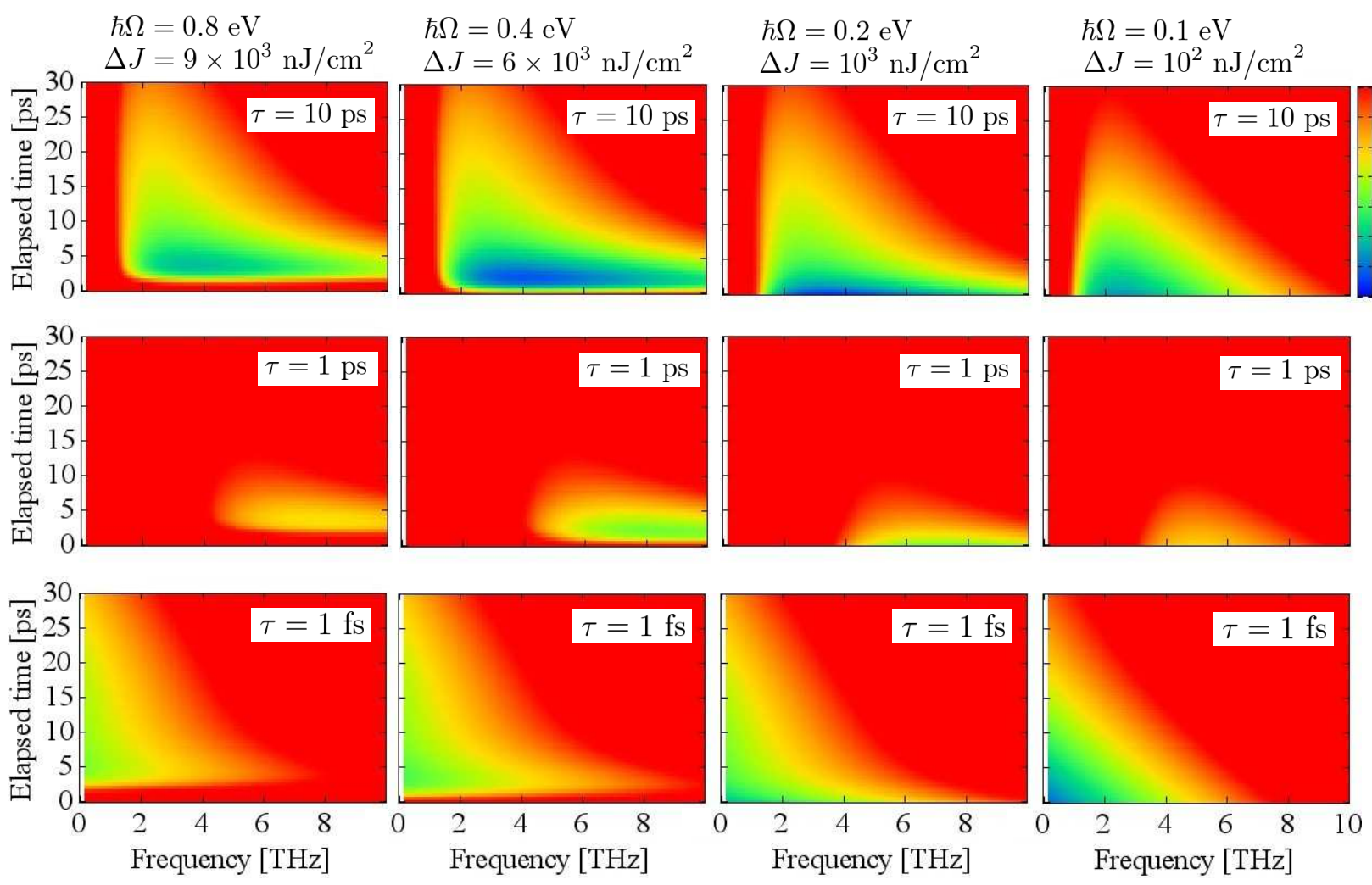

FIG. 3. The time-evolution of the dynamic conductivity in the THz range normalized to $e^{2} / 4 \hbar$ and cut off above zero. The columns from left to right correspond to cases with $\hbar \Omega=0.8,0.4,0.2$, and $0.1 \mathrm{eV}$ and $\Delta J=6 \times 10^{3}, 1 \times 10^{3}$, and $1 \times 10^{2}$ $\mathrm{nJ} / \mathrm{cm}^{2}$, respectively, whereas the rows from top to bottom correspond to cases with $\tau=10 \mathrm{ps}, 1 \mathrm{ps}$, and 1 fs, respectively.

drop in the region of photon energy below $0.25 \mathrm{eV}$, caused by the second and third factors.

The effect of the Pauli blocking depends strongly on the pulse photon energy. For $\hbar \Omega=0.8 \mathrm{eV}$, the changes in the quasi-Fermi level and carrier temperature were negligible for any pulse fluence in consideration when the Pauli blocking was taken into account. On the contrary, for $\hbar \Omega=0.1 \mathrm{eV}$, the threshold fluence changed $1.3-1.8$ times larger depending on the frequency, and the quasiFermi level achievable became smaller.

As has been discussed in Refs. 5 and 8 , the cooling in the case of low pulse photon energy occurs because the initial photoexcitation can give the lower energy to carriers than in thermal equilibrium. In fact, the energy of photogenerated carriers, $\hbar \Omega / 2$, is lower than the average energy of carriers in intrinsic graphene at room temperature, $\mathcal{E} / n \approx 0.0567 \mathrm{eV}$, when $\hbar \Omega<0.13 \mathrm{eV}$. Besides, the cooling after the heating by the photoexcitation (albeit small) can also occur as seen in Figs. 1(a)(c). This is associated with the discrete nature of OP emission/absorption which cannot exactly bring the carrier temperature to the lattice temperature. The latter is to be accomplished via the acoustic phonon scattering and/or radiative generation/recombination with substantially longer time scale.

\section{B. Time-Evolution of Dynamic Conductivity in Cases of High- and Low-Quality Graphene}

The dynamic conductivity in Eq. (5) depends primarily on the carrier distribution, i.e., the carrier concentration, as well as the momentum relaxation time $\tau$. Since the photoexcitation not only leads to the population inversion but also to the increase in the carrier concentration, the negative dynamic conductivity arises if the increase in the absolute value of the interband conductivity overcomes the increase in the Drude absorption in Eq. (5). For the realization of low threshold of negative dynamic conductivity in the $\mathrm{THz}$ range, it is necessary to suppress the Drude absorption. This can be done by using either good-quality graphene such that $\omega \tau \geq 1$ or high-quality graphene such that $\omega \tau \ll 1$. As can be seen in Eq. (5), in the former case, the high-frequency "cut-off" of the Drude conductivity occurs. In the latter case, the Drude conductivity ceases in the entire frequency range.

Figure 3 illustrates the time-evolution of normalized dynamic conductivity $\operatorname{Re} \sigma_{\omega} /\left(e^{2} / 4 \hbar\right)$ in the $\mathrm{THz}$ range with different pulse photon energy and momentum relaxation time. The pulse fluence for each pulse photon energy was chosen so that sufficiently large negative dynamic conductivity is obtained. It demonstrates that the negative dynamic conductivity can be achieved in the 

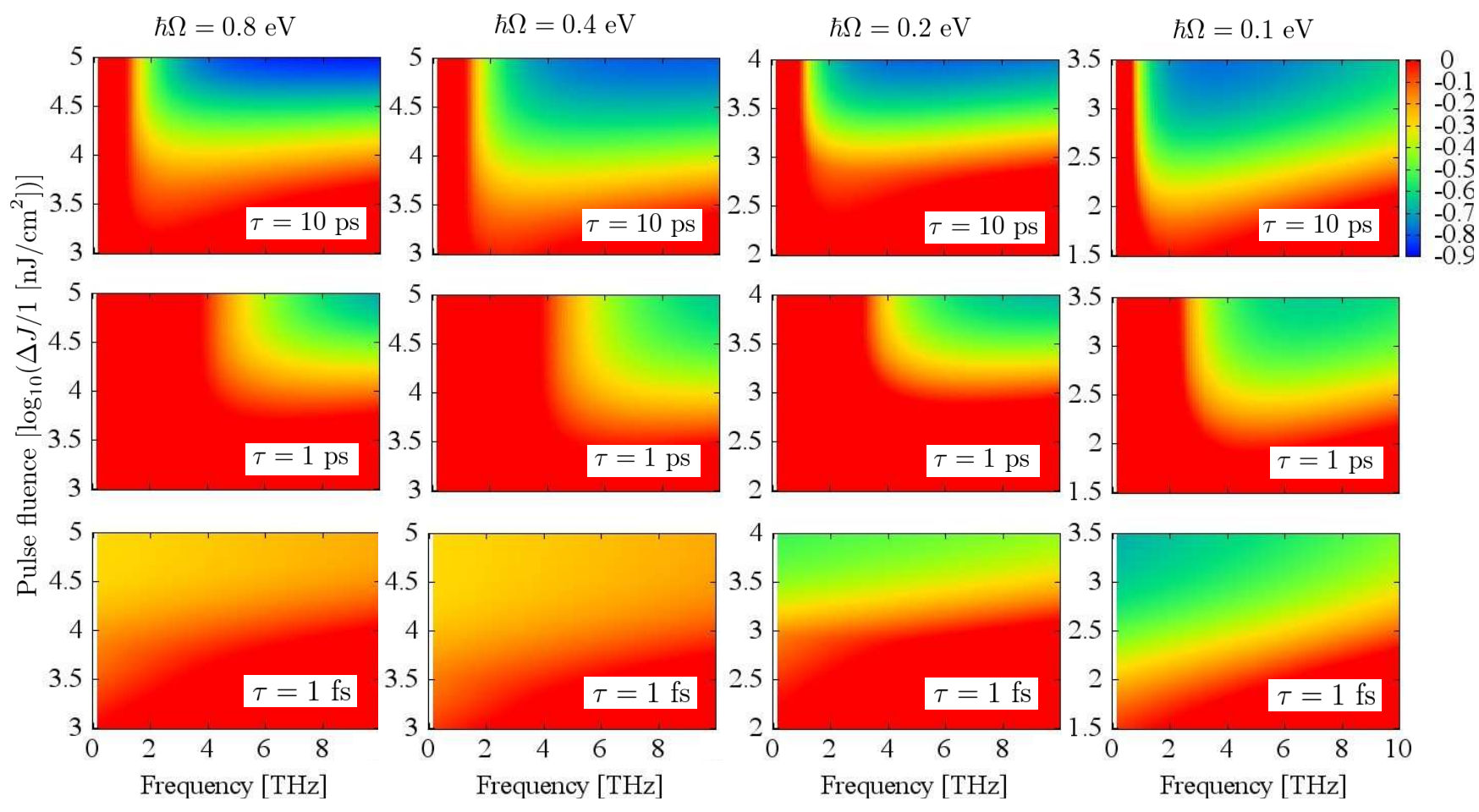

FIG. 4. The maximum negative value of the dynamic conductivity in its time duration as a function of frequency and pulse fluence. The columns from left to right correspond to cases with $\hbar \Omega=0.8,0.4,0.2$, and $0.1 \mathrm{eV}$, respectively, whereas the rows from top to bottom correspond to cases with $\tau=10 \mathrm{ps}, 1 \mathrm{ps}$, and $1 \mathrm{fs}$, respectively.

THz range with sufficiently high pulse fluence, and that the same level of negative dynamic conductivity can be achieved with much lower pulse fluence for lower pulse photon energy. It also shows that its magnitude, frequency range, and time duration depend strongly on the momentum relaxation time.

For $\tau=10 \mathrm{ps}$, the negative dynamic conductivity occurs down to $1 \mathrm{THz}$ and extends to more than $10 \mathrm{THz}$, and it duration is $5-30$ ps depending on the frequency. The duration is longer for lower frequency as is naturally understood from the relaxation of the quasi-Fermi level shown in Fig. 1. For $\tau=1$ ps, the negative dynamic conductivity occurs between 4 and $10 \mathrm{THz}$ with smaller magnitude and much shorter duration. This indicates that the high quality of graphene having longer momentum relaxation time is preferable. As can be seen in Fig. 3, the minimum frequency for the negative dynamic conductivity is primarily determined by the momentum relaxation time, although it slightly shifts to the lower side in case of the lower pulse photon energy.

On the contrary, for $\tau=1 \mathrm{fs}$, the negative dynamic conductivity occurs in the low frequency region below $6 \mathrm{THz}$. Note, however, that the meaning of the "timedependent dynamic conductivity" is valid only if its variation is slower than the frequency under consideration, approximately around or above a few hundred $\mathrm{GHz}$ in our case. This means we can have the negative dynamic conductivity down to a few hundred GHz. The upper limit of the frequency range having the negative dynamic conductivity in this case is smaller than in the case where $\omega \tau \ll 1$. This is because the Drude conductivity in this case is almost constant in the $\mathrm{THz}$ range, whereas it vanishes due to the high-frequency "cut-off" in the latter case.

Our calculation showed that even for $\hbar \Omega=0.1 \mathrm{eV}$ the threshold momentum relaxation time of the negative dynamic conductivity is $\tau=5 \mathrm{fs}$, and above it the dynamic conductivity does not occur in the $\mathrm{THz}$ range, no matter how large the pulse fluence is. This threshold roughly corresponds to the value at which the Drude term in Eq. (5) at zero-frequency limit exceeds the largest negative value of the interband contribution, i.e., $\tau \sim(\pi / 8 \log 2)\left(\hbar / k_{B} T_{l}\right)$.

Figure 4 shows the maximum negative value of the dynamic conductivity in its time duration as a function of frequency and pulse fluence with different pulse photon energy and different momentum relaxation time. It illustrates that the threshold pulse fluence as well as the pulse fluence needed to obtain the same value of the negative dynamic conductivity are decreased in several orders of magnitude for any frequency as the pulse photon energy is decreased. Comparing the cases with $\tau=10$ and 1 ps, it is seen that the decrease in the momentum relaxation time causes rather large increase in the minimum frequency for the negative dynamic conductivity and the increase in the threshold pulse fluence for frequency near 
the minimum, although the latter for frequency sufficiently above the minimum is insignificant due to the high-frequency "cut-off" of the Drude conductivity. As mentioned above already, the minimum frequency for the negative dynamic conductivity shifts to the lower side for the lower pulse photon energy. In fact, the frequency can be down to about $700 \mathrm{GHz}$ with $\hbar \Omega=0.1 \mathrm{eV}$ and $\tau=10$ ps, whereas it is around $1.1 \mathrm{THz}$ with $\hbar \Omega=0.8 \mathrm{eV}$ and $\tau=10 \mathrm{ps}$, demonstrating the superiority of the pumping by lower photon energy. For $\tau=1 \mathrm{fs}$, the threshold pulse fluence for each photon energy is on the same order in comparison with that for $\tau=10 \mathrm{ps}$.

Also, it should be noted that the maximum saturates below the "fundamental limit" (i.e., $-e^{2} / 4 \hbar$ ) for very high pulse fluence. For example, for $\tau=10 \mathrm{ps}$ and $\hbar \Omega=$ $0.1 \mathrm{eV}$, it saturates with the value $\operatorname{Re} \sigma_{\omega} /\left(e^{2} / 4 \hbar\right) \approx-0.4$ at $f=1 \mathrm{THz}$ and $\approx-0.8$ at $f=2 \mathrm{THz}$. The reason for this is that, for very high pulse fluence, the increase in the Drude absorption due to the increase in the carrier concentration exceeds the interband contribution even though very high quasi-Fermi level is obtained, and the maximum negative value of the dynamic conductivity corresponding to the saturation value is reached at later time after the relaxation of the carrier concentration. The Pauli blocking also takes part in the saturation in cases of lower photon energies. The saturation values for $\tau=1$ fs are smaller than those for $\tau=10 \mathrm{ps}$, especially, about 3 times smaller with $\hbar \Omega=0.4$ and $0.8 \mathrm{eV}$. Again, this is due to the nonzero, almost-constant value of the Drude conductivity in the $\mathrm{THz}$ range in the limit $\omega \tau \ll 1$.

\section{CONCLUSIONS}

We studied the population inversion and negative dynamic conductivity in intrinsic graphene upon pulse photoexcitation with the pulse photon energy in $0.1-0.8 \mathrm{eV}$ and their dependences on the pulse fluence. We showed that their threshold pulse fluence can be lowered by two orders of magnitude with the lower pulse photon energy. This is attributed to the inverse proportionality of the photogenerated carrier concentration to the photon energy, as well as to the weaker carrier heating which results in the denser accumulation of carriers in the low energy region and in the suppression of recombination via interband OP emission. We also showed that the negative dynamic conductivity takes place in the long relaxation time limit $\omega \tau \geq 1$, where the Drude absorption in the $\mathrm{THz}$ range becomes small by the high-frequency "cutoff", and in the very short relaxation time limit $\omega \tau \ll 1$, where it ceases in the entire frequency range. We demonstrated that the pulse excitation by lower pulse photon has both lower threshold pulse fluence and the lower minimum frequency for the negative dynamic conductivity down to about $700 \mathrm{GHz}$ with $\hbar \Omega=0.1 \mathrm{eV}$ and $\tau=10$ ps.

\section{ACKNOWLEDGMENTS}

This work was supported by JSPS Grant-in-Aid for Specially Promoted Research.
* a-satou@riec.tohoku.ac.jp

1 K. S. Novoselov, A. K. Geim, S. V. Morozov, D. Jiang, M. I. Katsnelson, I. V. Grigorieva, S. V. Dubonos, and A. A. Firsov, Nature 438, 197 (2005).

2 A. K. Geim and A. H. MacDonald, Physics Today 60, 35 (2007).

3 F. Bonaccorso, Z. Sun, T. Hasan, and A. C. Ferrari, Nature Photon. 4, 611 (2010).

4 V. Ryzhii, M. Ryzhii, and T. Otsuji, J. Appl. Phys. 101, 083114 (2007).

5 V. Ryzhii, M. Ryzhii, and T. Otsuji, Phys. Stat. Sol. (c) 5, 261 (2008).

${ }^{6}$ V. Ryzhii, M. Ryzhii, A. Satou, T. Otsuji, A. A. Dubinov, and V. Ya. Aleshkin, J. Appl. Phys 106, 084507 (2009).

7 A. Satou, T. Otsuji, and V. Ryzhii, Jpn. J. Appl. Phys. 50, 070116 (2011).

8 V. Ryzhii, M. Ryzhii, V. Mitin, A. Satou, and T. Otsuji, Jpn. J. Appl. Phys. 50, 094001 (2011).

9 V. Ryzhii, M. Ryzhii, V. Mitin, and T. Otsuji, J. Appl. Phys. 110, 094503 (2011).

10 V. Ryzhii, V. Mitin, M. Ryzhii, N. Ryabova, and T. Otsuji, Appl. Phys. Exp. 1, 063002 (2008).

11 V. Ryzhii, N. Ryabova, M. Ryzhii, N. V. Baryshnikov, V. E. Karasik, V. Mitin, and T. Otsuji, Optoelectronics Review 20, 15 (2012).
12 L. Vicarelli, M. S. Vitiello, D. Coquillat, A. Lombardo, A. C. Ferrari, W. Knap, M. Polini, V. Pellegrini, and A. Tredicucci, Nature Mat. 11, 865 (2012).

13 T. Otsuji, S. A. Boubanga Tombet, A. Satou, H. Fukidome, M. Suemitsu,E. Sano, V. Popov, M. Ryzhii, and V. Ryzhii, J. Phys. D: Appl. Phys. 45, 303001 (2012).

14 H. Karasawa, T. Komori, T. Watanabe, H. Fukidome, M. Suemitsu, V. Ryzhii, and T. Otsuji, J. Infrared Milli. Thrz. Waves 32, 655 (2011).

15 S. Boubanga-Tombet, S. Chan, T. Watanabe, A. Satou, V. Ryzhii, and T. Otsuji, Phys. Rev. B 85, 035443 (2012).

16 D. Sun, Z.-K. Wu, C. Divin, X. Li, C. Berger, W. A. de Heer, P. N. First, and T. B. Norris, Phys. Rev. Lett. 101, 157402 (2008).

17 J. M. Dawlaty, S. Shivaraman, M. Chandrashekhar, F. Rana, and M. G. Spencer, Appl. Phys. Lett. 92, 042116 (2008).

18 M. Breusing, C. Ropers, and T. Elsaesser, Phys. Rev. Lett. 102, 086809 (2009).

19 K. M. Dani, J. Lee, R. Sharma, A. D. Mohite, C. M. Galande, P. M. Ajayan, A. M. Dattelbaum, H. Htoon, A. J. Taylor, and R. P. Prasankumar, Phys. Rev. B 86, 125403 (2012).

20 A. Satou, T. Otsuji, V. Ryzhii, and F. T. Vasko, Proc. PIERS in Kuala Lumpur, 486 (2012). 
21 Although more elaborated model based on the system of the Bloch equations which takes into account quantum many-particle effects has been developed in the literature [for instance, see E. Malic, T. Winzer, E. Bobkin, and A. Knorr, Phys. Rev. B 84, 205406 (2011)], the quasiclassical approach used here suffices for the qualitative analysis of the threshold behavior of the population inversion and negative dynamic conductivity.

${ }^{22}$ L. A. Falkovsky and A. A. Varlamov, Eur. Phys. J. 56, 281 (2007). 